\title{
Vertical bone regeneration with synthetic biomimetic calcium phosphate onto the calvaria of rats
}

\begin{abstract}
Alain Hoornaert DDS, $\mathrm{PhD}^{1,2}$, Yassine Maazouz $\mathrm{PhD}^{3}$, David Pastorino $\mathrm{PhD}^{3}$,
\end{abstract} Carlos Aparicio DDS, PhD ${ }^{3}$, Gonzague de Pinieux MD, PhD ${ }^{1,4}$,

Borhane H. Fellah DVM, PhD ${ }^{5}$, Maria-Pau Ginebra PhD ${ }^{6}$, Pierre Layrolle PhD ${ }^{1, *}$

1) Inserm, UMR 1238, PHY-OS, Bone sarcomas and remodelling of calcified tissues, Faculty of Medicine, University of Nantes, Nantes, France.

2) CHU Nantes, Faculty of Dental Surgery, Nantes, France.

3) Mimetis Biomaterials, Barcelona, Spain.

4) CHU Tours, Department of AnatomoPathology, Tours, France.

5) ONIRIS, Veterinary School of Nantes, Centre for investigation on pre-clinical research, Nantes, France.

6) Biomaterials, Biomechanics and Tissue Engineering Group, Department of Materials Science and Metallurgical Engineering, Universitat Politècnica de Catalunya, Barcelona, Spain.

* Corresponding author: Prof. Pierre Layrolle, PhD, Director of Research, Inserm UMR 1238, PHY-OS, Faculty of Medicine, 1 rue Gaston Veil, 44035 Nantes, France. Tel: +33 (0)2 726411 43; Fax: +33 (0)2 404128 60; Email: pierre.layrolle@inserm.fr

Date of original submission to the journal Tissue Engineering Part C: 4/09/2018

Date of revision: $23 / 10 / 2018$ 
Statement of significance

This work reports a new bone substitute made of precipitated apatite crystals that resemble in composition and crystallinity to the mineral phase of bone. The bone regeneration capacity of this synthetic biomimetic calcium phosphate (SBCP) was studied by using an original model of vertical bone regeneration with cups on the calvaria of rats. After 4 weeks, a significantly higher bone growth was found for SBCP compared to deproteinized bovine bone matrix (DBBM) and empty controls. This rapid vertical bone regeneration indicated that this new biomaterial is particularly interesting for filling bone defects in oral surgery. 


\section{Abstract}

Bone regeneration is often required to provide adequate oral rehabilitation before dental implants. Vertical ridge augmentation is the most challenging of all situations and often requires the use of autologous bone grafting. However, autologous bone grafting induces morbidity and the harvestable bone is limited in quantity. Alternatives to autologous bone grafting include bovine bone derived biomaterials which provide good clinical results and synthetic bone substitutes that still fail to provide a reliable clinical outcome. Synthetic biomimetic calcium phosphate biomaterials, consisting of precipitated apatite crystals that resemble in composition and crystallinity the mineral phase of bone, arise as an alternative to both bovine bone and the current sintered bone substitutes. This study aims at comparing the vertical bone regeneration capacity of the synthetic biomimetic calcium phosphate (SBCP, MimetikOss, Mimetis Biomaterials) with a deproteinized bovine bone matrix (DBBM, Bio-Oss ${ }^{\circledR}$, Geistlich Biomaterials) on the calvaria of rats. In order to model vertical bone augmentation, hemispherical cups were filled with the two types of biomaterial granules and implanted onto the skull of rats while empty cups were used as controls. After 4 and 8 weeks of healing, bone growth was determined by microcomputed tomography and histomorphometry. After 4 weeks of implantation, a significantly higher bone growth was found in the case of SBCP compared to DBBM and left empty controls. At 8 weeks, no statistically significant differences were found between the two bone substitutes. These results are promising since vertical bone regeneration was faster in the case of SBCP than for DBBM.

Keywords: Vertical bone regeneration, synthetic biomimetic calcium phosphate, deproteinized bovine bone Matrix, Hydroxyapatite, cups, calvaria, rats. 


\section{Introduction}

Bone augmentation is often required prior to the placement of dental implants, especially in cases where both vestibular and lingual alveolar bone suffered bone resorption after tooth extraction. In this context, one of the major challenges in dental surgery is vertical bone augmentation [1,2] which has proven to be highly unpredictable even for experienced surgeons [3]. In order to tackle this problem, oral surgeons use bone grafts that can ensure a predictable and reliable clinical outcome. For augmentation of the alveolar bone crest, autologous bone remains the gold standard due to its osteogenic properties and similarity with the host tissue. However, autologous bone has drawbacks such as morbidity at the donor site, limited quantity and high resorption rate $[4,5]$. The use of bone graft substitutes (BGS) in bone regeneration has proven to be efficient in some dental indications, such as sinus lift and alveolar ridge preservation after tooth extraction $[6,7]$. In these particular sites, the BGS is surrounded by several bone walls, protecting granulated BGS from potential migration and movements thus favouring bone regeneration. However, none of the commercially available dental BGS has demonstrated to be reliable in vertical bone augmentation of the alveolar crest $[8,9]$ even if some studies state that deproteinized bovine bone matrix (DBBM) is a good candidate for vertical ridge augmentation [7].

Despite some chemical similarities with the mineral of bone, synthetic calcium phosphate bioceramics, such as hydroxyapatite (HA) and beta-tricalcium phosphate ( $\beta$-TCP), present poor bone regenerative properties in large defects and variable resorption rate [10]. The reasons may be related to the high sintering temperatures of bioceramics (e.g. 800-1200 ${ }^{\circ} \mathrm{C}$ ), which confers physicochemical features differing drastically from the mineral phase of bone, which is naturally precipitated from body fluids at body temperatures. Xenografts, such as deproteinized bovine bone matrix (DBBM) have proven their efficacy in a wide range of dental indications but present low bone remodelling capacity over time $[11,12]$. Furthermore, animal-derived products may potentially carry risks of immunological rejection and disease transmission such as bovine spongiform encephalopathy [13]. 
Synthetic Biomimetic Calcium Phosphate (SBCP) is a very attractive alternative due to their synthesis route. SBCP presents many similarities with natural bone [14]. SBCP, which is obtained by precipitation in aqueous media at low temperature, is composed of a calciumdeficient hydroxyapatite (CDHA) and have low crystallinity resembling the mineral phase of bone. These biomimetic materials, e.g. in the form of calcium phosphate cement, have been primarily used as bone fillers, although the absence of macro-porosity in cements prevents bone ingrowth and limits the regenerative capacity. Granules of 0.2-1 mm in size present an inter-granular porosity that facilitates body fluid permeability, cell colonization, vascularization and bone tissue ingrowth [15]. There is a limited number of studies comparing the in vivo performance of DBBM and SBCP granules in a model of vertical bone augmentation [1]. In order to mimic the clinical situation of vertical bone augmentation, a preclinical model consisting of hemispheric cups filled with biomaterial granules on the calvarias of rats is proposed here.

This study aims at comparing the vertical bone regeneration capacity of a synthetic biomimetic calcium phosphate (SBCP, MimetikOss, Mimetis Biomaterials, Spain) and a deproteinized bovine bone matrix (DBBM, Bio-Oss ${ }^{\circledR}$, Geistlich Biomaterials, Switzerland) to promote vertical bone formation onto the calvaria of rats. Hemispherical cups were filled with the two types of granules and implanted on the skull of rats while empty cups were used as controls. After 4 and 8 weeks of healing, the biomaterial content and bone growth were determined by microcomputed tomography and histomorphometry.

\section{Materials and methods}

\section{Hemispherical cups}

Hemispherical hollow cups were drawn by computer-aided design and milled from polymethyl methacrylate (PMMA) resin discs (TEMP Basic, Zirkonzahn, Italy). PMMA was chosen because of its biocompatibility, radiolucent characteristics and solubility in solvents during histological processing. As shown in Figure 1, the hemispheric cups had an internal diameter of $6 \mathrm{~mm}$, a wall of $1 \mathrm{~mm}$ and an outer dimeter of $7 \mathrm{~mm}$ as well as fixation reliefs to ensure stability once placed onto the calvaria of rats. 


\section{Bone graft substitutes}

Two types of bone graft substitutes (BGS) were used to fill the cups: deproteinized bovine bone matrix (DBBM, Bio-Oss ${ }^{\bullet}$, Geistlich Biomaterials, Switzerland) in granular form with sizes between 0.25 and $1 \mathrm{~mm}$ and synthetic biomimetic calcium phosphate (SBCP, MimetikOss, Mimetis Biomaterials, Spain) in granular form with sizes between 0.2 and 1 $\mathrm{mm}$. Both BGS were provided in vials sealed into blisters, sterilized by gamma irradiation and used following the instructions for use.

\section{Physico-chemical characterization of bone graft substitutes}

The microstructure of the BGS was observed by scanning electron microscopy (Neon 40, Zeiss, Germany) operating at $5 \mathrm{kV}$ after metallization with AuV-sputter coating (K950X, Emitech, US). Mercury intrusion porosimetry (MIP, AutoPore IV, Micromeritics, USA) was performed to determine the pore entrance size distribution by using a specific $3 \mathrm{~cm}^{3}$ powder holder. The intra-granular porosity was defined as micropores $<10 \mu \mathrm{m}$ in size on granules while the inter-granular porosity corresponded to the spaces between packed granules with a pore size $>10 \mu \mathrm{m}$. The specific surface area (SSA) was evaluated by nitrogen adsorption using the Brunauer-Emmett-Teller theory (Micromeritics, ASAP 2020, USA). Samples were also analysed by ATR-FTIR (Nicolet 6700, Thermo Scientific). X-ray diffraction (XRD) was performed on the finely ground granules of both DBBM and SBCP. Frozen dried sample of bovine bone (obtained from a butcher) was used to compare the composition of BGS to native bone tissue in terms of chemical composition and crystalline phases. XRD analyses were performed using a powder diffractometer (D8 Advance, Bruker, Germany) with Bragg-Brentano geometry equipped with a germanium monochromator and a Cu Ka source operating at $40 \mathrm{kV}$ and $40 \mathrm{~mA}$. Data sets were collected with a step size of $0.019^{\circ}$ in $2 \theta$ and a counting time of $1 \mathrm{~s}$ per step. The diffraction patterns were compared with files from the Joint Committee on Powder Diffraction Standards for $\alpha$-TCP (JCPDS No. 9-348), $\beta$-TCP (JCPDS No. 9-169) and hydroxyapatite (HA; JCPDS No. 9-432). Phase quantification was performed by comparing the ratios of the area for the most intense peak using the XRD analysis software (EVA, Bruker, Germany). 


\section{Study Design}

All experimental procedures and protocols were reviewed and approved by the local animal care and use ethics committee (Reference of the study CEEA 2012.188 accepted on 17/01/2013). The European regulation on the use and care of experimental animals was followed carefully. Fifty-two Wistar albino rats (strain: Wistar, Rj:Han, adult, male, average body weight of $150 \mathrm{~g}$ ) were purchased from a professional stock breeder (Janvier Labs, Le Genest Saint Isle, France). Three animals were placed per cage with pelleted food and water in a temperature-controlled room with 12 hours artificial day/night cycle. Animals were acclimatised at the Experimental Therapeutic Unit, Faculty of Medicine of Nantes, for a minimum of 10 days prior to surgery. The study design consisted of 3 groups: empty cups, cups filled with DBBM granules or with SBCP granules; and 3 time points: 1 day, 4 weeks and 8 weeks ( $n=6$ per group). At 1 day, the empty condition was not considered since the granules were not present for histomorphometry analysis. The total number of rats was 48 , plus 4 spares, giving a total of 52 animals.

\section{Surgical procedure}

Each rat was placed on the ventral position under general anaesthesia by inhalation with a mask of $3 \%$ isofluorane (Forane ${ }^{\circledR}$, Baxter Healthcare Corp., USA) in air at a flowrate of 1 L/min. The head was shaved and disinfected with $10 \%$ povidone iodine solution (Betadine ${ }^{\bullet}$ Scrub) and sterile gauzes. Local anaesthesia was performed by subcutis injection of adrenaline articaine hydrochloride (0.2 ml, Alphacaine SP, Dentsply, France). Pre- and post-operative analgesia was provided by intramuscular injection of buprenorphine (30 $\mu \mathrm{l} / \mathrm{kg}, 2$ times/day for 3 days, Buprecare, Axience, Pantin, France). The animals were identified with numbered ear tags. The bone calvaria was exposed by a lateral incision and smooth dissection. The periosteum was incised and detached from the bone calvaria. To ease the filling of the cup, the BGS granules were hydrated with physiological saline and gently packed in the hemispheric cups. A cup, either left empty or filled with the BGS granules, was placed onto the bone calvaria under the skin. The skin was carefully closed with non-absorbable polyamide 4/0 sutures (Peters Surgicals, Bobigny, France). After 1 day, 4, and 8 weeks of implantation, the animals were euthanized 
by prolonged inhalation of carbon dioxide gas. The site was dissected and examined for signs of tissue necrosis, inflammation or infection. The calvaria was cut using a circular diamond saw mounted on a dental hand piece (NM3000, Nouvag, Switzerland). Immediately after dissection, samples were fixed in 10 volumes of neutral $4 \%$ formaldehyde (Microm Microtech, France) and stored at $4{ }^{\circ} \mathrm{C}$ for a minimum of 3 days.

\section{Microcomputed tomography}

Microcomputed tomography (micro-CT) analysis was performed on calvaria after 1 day, 4 weeks and 8 weeks using a high-resolution X-ray micro-CT system for small-animal imaging (micro-CT 1076, SkyScan, Kontich, Belgium). As the two biomaterials have different size and shape, day 1 implantation was used for determination of BV+MV/TV (\%) without any degradation of the biomaterial or bone regeneration in the cups. All samples were scanned using the same parameters (pixel size $9 \mu \mathrm{m}, 50 \mathrm{kV}, 0.5 \mathrm{~mm} \mathrm{Al}$ filter and $0.8^{\circ}$ of rotation step). Three-dimensional reconstructions were made using a software (CTVOX, Skyscan, Belgium) to evaluate the filling capacity of the BGS in the cup. Bone and biomaterial volume over total volume $(\mathrm{BV}+\mathrm{MV}) / \mathrm{TV}$ were determined in the cup owing to a software allowing to finely select the threshold (CTAN, Skyscan, Belgium) on 3 randomly selected samples for each time point.

\section{Histology and histomorphometry}

The samples were decalcified with $5 \%$ ethylene diamine tetra acetic acid (EDTA) and $0.2 \%$ paraformaldehyde in phosphate-buffered saline (PBS) for $96 \mathrm{~h}$ using a microwave automate (KOS Microwave, HistoStation, Milestone Medical, USA). The samples were then rinsed with tap water and dehydrated in ascending series of 80, 95 and $100 \%$ ethanol baths, and finally in butanol for $30 \mathrm{~min}$ (Automated dehydration station, Microm Microtech, France). After dehydration, the PMMA cup was dissolved in acetone. The samples were then immersed in liquid paraffin at $56{ }^{\circ} \mathrm{C}$ (Histowax, Histolab Products $A B$, Sweden) and embedded at $-16{ }^{\circ} \mathrm{C}$. Blocks were cut by using a standard microtome (RM2250, Leica, Germany). Thin histological serial sections (3-5 $\mu \mathrm{m})$ were performed perpendicular to the calvaria in the middle of the cup. The slices were mounted on microscope glass slides (Polysine, Thermo Scientific, Germany) and stained with Masson's 
trichrome using an automated staining station (Microm Microtech). This staining combines haematein for cell nuclei (purple/black), fushin Ponceau for cytoplasm, muscle and erythrocytes (red) and light green solution for collagen (green/blue). The stained slices were scanned (NanoZoomer 2.0RS, Hamamatsu Corp. Japan) and observed with the virtual microscope (NDP view software, Hamamatsu Corp). The parameters quantified in the region of interest (Figure 1) by histomorphometric analysis (Image J, National Institute of Health, USA) were the area percentages of biomaterial (MS/TS), bone (BS/TS) and residual tissues (RS/TS). The vertical bone regeneration in the cup was determined by calculating the percentage of newly formed bone in the free space, defined as follows:

$\%$ Bone surface in free space $=(B S /(B S+R S)) \times 100$

where:

BS is the bone surface in the region of interest

MS is the biomaterial surface in the region of interest

TS is the total surface of the region of interest

$\mathrm{RS}$ is the residual tissue surface of the region of interest

\section{Statistical analysis}

Based on a statistical power calculation, 6 animals were used per group. Data are presented as average \pm standard error of the mean. Statistical differences were determined using one-way ANOVA with Tukey's post hoc tests using Minitab 16 software (Minitab, Inc., USA). Statistical significance was considered for $\mathrm{p}$ value $<0.05$.

\section{Results}

\section{Physico-chemical properties of the bone graft substitutes}

The morphology and microstructure of the two bone graft substitutes (BGS) were observed by scanning electron microscopy (SEM). As illustrated in Figure 2, the Deproteinized Bovine Bone Matrix (DBBM) granules showed an irregular shape with one large and two short dimensions, while the synthetic biomimetic calcium phosphate (SBCP) 
granules appeared spherical. At high magnification, the DBBM surface exhibited aligned micropores whereas needle-like crystals were observed on the SBCP surface.

The pore size distribution was measured by mercury intrusion porosimetry. Both BGS presented a bimodal pore entrance size distribution (Figure 3). The left peak, corresponding to the smallest pore entrance diameters, was considered to represent the micro/nano porosity of the granules themselves (intra-granule porosity). These peaks were in the range 0.01-0.03 $\mu \mathrm{m}$ and $0.1 \mu \mathrm{m}$ for DBBM and SBCP, respectively. The right peak, at higher entrance pore sizes, corresponded to the spaces between the granules (intergranular porosity). Peaks had a maximum value at $200 \mu \mathrm{m}$ and $150 \mu \mathrm{m}$ for DBBM and SBCP, respectively. The peak of inter-granular macro-porosity exhibited by SBCP was wider than the one of DBBM. Quantification of the corresponding pore volumes led to the determination of the intra-granular microporosity $(<10 \mu \mathrm{m})$, inter-granular porosity $(>10$ $\mu \mathrm{m}$ ) and total porosity, as presented in Table 1. DBBM was found to have a slightly higher intra-granular micro-porosity than SBCP, while the inter-granular porosity of SBCP was substantially higher. SBCP presented a higher total porosity than DBBM. The SSA, as measured by Nitrogen adsorption is also reported in Table 1. The SSA of DBBM was higher than that of SBCP by an order of magnitude.

The FTIR spectra of the two biomaterials, together with that of the frozen dehydrated bovine bone, are shown in Figure 4a. Both DBBM and SBCP exhibited the phosphate bands of hydroxyapatite, similarly to what was found in dehydrated bone. In natural bone, these phosphate bands coexist with others assigned to the organic compounds, namely collagen and other extracellular matrix proteins with their characteristic amide bands. Interestingly, the presence of $\mathrm{HPO}_{4}{ }^{-}$was noted on the $3 \mathrm{FTIR}$ spectra. This $\mathrm{HPO}_{4}^{-}$band at $960 \mathrm{~cm}^{-1}$ is characteristic of calcium deficient hydroxyapatite, group of which sintered calcium phosphate are deprived. The XRD patterns of the different biomaterials are shown in Figure $4 \mathrm{~b}$. DBBM consisted of a hydroxyapatite phase with a comparable crystallinity as found in natural bone. SBCP was composed of two crystalline phases, calcium deficient HA and $\beta$-TCP in a weight proportion of $80 \%$ and $20 \%$, respectively. 


\section{Comparison of vertical bone regeneration on the calvaria of rats}

After surgery, all animals exhibited normal clinical symptoms and the sutures were checked for correct healing. Rats were all feeding themselves correctly after a few hours as analgesia was maintained during the first 72 hours. No major pain symptoms were observed after this period and animals gained weight normally. At sacrifice after 4 and 8 weeks, no signs of infections or tissue necrosis were noticed.

Microcomputed tomography (micro-CT) reconstructions are provided in Figure 5. Newly formed bone was not found in the empty cups after 4 and 8 weeks. In general, the granules were well packed and maintained into the cups, although some granules were found outside one day, 4 and 8 weeks after surgery. As reported in Table 2, the $(B V+M V) / T V$ calculations indicated a limited quantity of newly formed bone in the left empty cups after 4 and 8 weeks indicating the validity of this vertical bone augmentation model. One day after surgery, the cups appeared to be well-filled with granules for both types of biomaterials although the quantity was higher for SBCP than for DBBM due to the higher packing of spherical granules. The (BV+MV)/TV gradually increased from 1 day to 8 weeks for the DBBM. The (BV+MV)/TV of the SBCP first decreased from 1 day to 4 weeks before increasing to a similar value as DBBM after 8 weeks. Both groups of biomaterials filled cups had similar (BV+MV)/TV values after 8 weeks (no statistical differences). The two biomaterials had significantly higher BV+MV/TV values than the control left empty cups at 4 and 8 weeks $(p<0.05)$ although these differences may simply result from the filling of the cups with BGS. Indeed, it was not possible to differentiate between newly formed bone and biomaterial by using micro-CT due to their similar X-ray absorption levels.

Decalcified histology was employed to directly observe the tissues formed within the cups around biomaterial granules as micro-CT did not allow the distinction between bone and biomaterial with similar grey levels. A representative histology section of each condition at each time point is reported in Figure 6 at low magnification and at high magnification in Figures 7-9. When the cup was left empty, a very limited vertical bone growth was observed at both 4 and 8 weeks after surgery. The calvaria bone exhibited a vertical 
thickening with a newly formed non-woven bone that originated from the bone surface while most of the cup was filled with a necrotic tissue at 4 weeks (Figures 6 and 7). Thickening of the cortical bone was also observed at 8 weeks with mature lamellar bone covered by a thin vascularized fibrous tissue. Numerous necrotic cells occupied the rest of the left empty cups. In the case of DBBM, some bone formation was observed at 4 weeks in the "cortical" area originating from the periosteum (Figures 6 and 8). Woven bone was observed between the granules and a few blood vessels were distinguished. The "cup" area showed limited signs of bone formation at 4 weeks but was filled with newly formed bone at 8 weeks. The majority of DBBM granules were encapsulated in a vascularized fibrous tissue without macrophages or giant cells typical of a foreign body reaction. For cups filled with the SBCP granules, newly formed bone was observed in the cortical area with some fibro-vascular tissue at 4 weeks (Figures 6 and 9). The SBCP granules appeared surrounded by a mineralized collagen tissue with osteoblastic cells. Bone formation at 8 weeks in this area was complete and the granules were surrounded by lamellar bone close to the cortical bone and woven bone in the rest of the cup. Again, osteoblastic cells were observed around the SBCP granules with macrophages indicating a foreign body reaction. The cup area showed limited signs of bone formation at 4 weeks and good bone formation between granules at 8 weeks, as shown in Figure 6 and 9.

As shown in Figure 10, histomorphometry corroborated that SBCP presented superior bone formation capacity in the "cortical" area compared to DBBM or empty conditions at 4 weeks $(p=0.01)$. The density of bone in free space was statistically higher for SBCP as compared to DBBM at 4 weeks with a p-value of 0.01 . At 8 weeks, no statistically significant differences were observed between DBBM and SBCP, showing a percentage of bone between the biomaterial granules of $60.6 \pm 14.1 \%$ and $73.3 \pm 18.8 \%$, respectively. The values of MS/TS (Figure 10) obtained by histomorphometric analysis corroborated that SBCP granules pack better into the cup, although no statistically significant differences were observed between the two biomaterials $(p=0.09)$. The MS/TS value did not change over time for both types of biomaterial. 


\section{Discussion}

The use of novel biomimetic synthetic calcium phosphate bone substitute that resembles closely the mineral phase of bone is of high interest for bone regeneration. Biomimetic materials are the focus of many research teams since they are claimed to possess superior osteoconductive properties owing to their micro/nano porosity and their high specific surface area which is lower than DBBM but can still be considered as a high value as compared to other synthetic calcium phosphate bioceramics produced mainly by sintering at high temperatures $[16,17]$.

In this study, the bone regeneration capacity of this synthetic biomimetic calcium phosphate (SBCP) was studied by using an original model of vertical bone regeneration with hemispheric cups filled with biomaterial on the calvaria of rats. A similar model with hemispheric cups made of titanium has been reported in rabbits calvarium but has never been used on calvaria of rats [18-23]. These hemispheric domes provide with an immobilized space for vertical bone regeneration and mimics the clinical situation of alveolar bone augmentation where stable barrier membranes made of titanium reinforced polytetrafluorethylene membranes are used [24]. In place of titanium domes previously used in rabbits calvarium, we have selected PMMA hemispheric cups that have the advantages of being biocompatible, had sufficient stability and stiffness to maintain a bone space as well as offering the possibility of micro-CT analysis and direct processing with decalcified histology. However, this model of hemispheric cup has several limitations. In particular, the PMMA cups only allowed new blood vessels ingrowth from the calvaria bone while preventing the vascularization from the hypodermal tissue. Consequently, abundant necrotic tissue was observed in the cups. An interesting improvement of the model would consist of drilling holes in the hemispheric cup to permit the invasion of cells and blood vessels. PMMA is also a less osteoconductive material than titanium although bone conduction was observed on the inner walls of the cups filled with SBCP (Figure 6). Furthermore, both the stability of the cup and bone regeneration could be improved by preparing a circular groove with trephine on the calvaria bone of rats as described in previous studies $[18,19,22]$. 
The empty cup was considered as the negative control condition, although some bone growth was observed at 4 and 8 weeks, suggesting that the initial stimulation by scratching cortical bone stimulated a slight bone growth even in the case where no scaffolding was provided. This overgrowth of calvaria bone has also been reported in a rabbit model with BV/TV of $43.1 \pm 7.3$ and $29.8 \pm 6.8 \%$ at 1 and 3 months, respectively [20]. In our study, the BV/TV in empty was significantly lower than in the previous study with $1.36 \pm 0.42$ and $2.33 \pm 2.15 \%$ at 4 and 8 weeks, respectively (Table 2). These differences in bone overgrowth may be explained by the creation of a circular groove on rabbits calvarium while scratching of calvarias was only used in our model in rats.

Based on the hypothesis that the two tested biomaterials presented different initial packing abilities, the percentage of bone between granules was calculated by using a method described by Flautre et al. [25]. SBCP recorded a significantly higher value of bone in the free spaces at 4 weeks, while the difference was not significant at 8 weeks, as compared to DBBM. This result suggests that synthetic biomimetic calcium phosphate granules are able to conduct bone ingrowth faster than deproteinized bovine bone matrix granules. The shape of the granules, their micro structure and the higher total porosity can be partly responsible for the faster bone regeneration capacity of SBCP as compared to DBBM [17]. These results suggest that SBCP is superior to DBBM and that a possible faster mode of action makes this type of biomimetic calcium phosphate biomaterial a good candidate for their use in challenging dental indications such as vertical bone augmentation. Further studies are needed in a large animal model in a dental indication to further confirm the findings of the present study. Nevertheless, none of the condition indicated a complete filling of the cups with bone tissue. It will be of interest to conduct a similar experiment with bone marrow derived mesenchymal stem cells associated to the biomaterial in order to induce bone tissue formation, as previously reported [26]. Another limitation of our study concerns vascularization that is key to bone regeneration. The proportion of blood vessels at 4 and 8 weeks seems to be superior for SBCP than for DBBM but remained insufficient for a complete bone regeneration in the cups. 


\section{Conclusion}

Synthetic biomimetic calcium phosphate granules were evaluated as a good candidate for vertical bone augmentation. This new bone graft substitute outperformed DBBM in terms of bone formation rate since newly formed bone tissue was more abundant at 4 weeks of implantation for SBCP. Furthermore, owing to their physicochemical characteristics and packing features, the SBCP granules have a great potential for alveolar bone ridge augmentation.

\section{Acknowledgments}

The authors kindly acknowledge Joan Boix Sala for his substantial help in the fabrication and milling of the hemispherical hollow cups and Dr Maria Cristina Manzanares for her help regarding histological observation.

\section{Disclosure of interests}

Mimetis Biomaterials is a company that funded the study. Y. Maazouz, D. Pastorino, C. Aparicio and M.P. Ginebra are shareholders of the company. 


\title{
References
}

\author{
[1] T.L. Aghaloo, P.K. Moy, Which hard tissue augmentation techniques are the most \\ successful in furnishing bony support for implant placement? in: Int J Oral \\ Maxillofac Implants, 2007: 23(1):56-70. \\ [2] B.S. McAllister, K. Haghighat, Bone augmentation techniques, J. Periodontol. \\ (2007) 78(3): 377-96. \\ [3] M. Simion, S.A. Jovanovic, C. Tinti, S.P. Benfenati, Long-term evaluation of \\ osseointegrated implants inserted at the time or after vertical ridge augmentation, \\ Clin. Oral Impl. Res. 12 (2001) 35-45. \\ [4] L. Cordaro, D.S. Amadè, M. Cordaro, Clinical results of alveolar ridge augmentation \\ with mandibular block bone grafts in partially edentulous patients prior to implant \\ placement, Clin. Oral Impl. Res. 13 (2002) 103-111. \\ [5] L. Cordaro, F. Torsello, M. Tindara Miuccio, V. Mirisola di Torresanto, D. Eliopoulos, \\ Mandibular bone harvesting for alveolar reconstruction and implant placement: \\ subjective and objective cross-sectional evaluation of donor and recipient site up \\ to 4 years, Clin. Oral Impl. Res. 22 (2011) 1320-1326. \\ [6] A.R. Rissolo, J. Bennett, Bone grafting and its essential role in implant dentistry, \\ Dent Clin North Am. 42 (1998) 91-116. \\ [7] S.S. Jensen, H. Terheyden, Bone augmentation procedures in localized defects in \\ the alveolar ridge: clinical results with different bone grafts and bone-substitute \\ materials, Int J Oral Maxillofac Implants. 24 Suppl (2008) 218-236. \\ [8] M. Sanz, F. Vignoletti, Key aspects on the use of bone substitutes for bone \\ regeneration of edentulous ridges, Dent Mater. 31 (2015) 640-647. \\ [9] R. Lutz, F.W. Neukam, M. Simion, C.M. Schmitt, Long-term outcomes of bone \\ augmentation on soft and hard-tissue stability: a systematic review, Clin. Oral \\ Impl. Res. 26 Suppl 11 (2015) 103-122.
}


[10] J. Zhang, W. Liu, V. Schnitzler, F. Tancret, J.-M. Bouler, Calcium phosphate cements for bone substitution: chemistry, handling and mechanical properties, Acta Biomater. 10 (2014) 1035-1049.

[11] S. Milani, L. Dal Pozzo, G. Rasperini, C. Sforza, C. Dellavia, Deproteinized bovine bone remodeling pattern in alveolar socket: a clinical immunohistological evaluation, Clin. Oral Impl. Res. 27 (2016) 295-302.

[12]

P. Galindo-Moreno, P. Hernández-Cortés, F. Mesa, N. Carranza, G. Juodzbalys, M. Aguilar, et al., Slow resorption of anorganic bovine bone by osteoclasts in maxillary sinus augmentation, Clin Implant Dent Relat Res. 15 (2013) 858-866.

Y. Kim, A.E. Rodriguez, H. Nowzari, The Risk of Prion Infection through Bovine Grafting Materials, Clin Implant Dent Relat Res. 18 (2016) 1095-1102.

H. Guo, J. Su, J. Wei, H. Kong, C. Liu, Biocompatibility and osteogenicity of degradable Ca-deficient hydroxyapatite scaffolds from calcium phosphate cement for bone tissue engineering, Acta Biomater. 5 (2009) 268-278.

B. Ben-Nissan, Advances in Calcium Phosphate Biomaterials, Springer Science \& Business, Berlin, Heidelberg, 2014.

R.J. Miron, A. Sculean, Y. Shuang, D.D. Bosshardt, R. Gruber, D. Buser, et al., Osteoinductive potential of a novel biphasic calcium phosphate bone graft in comparison with autographs, xenografts, and DFDBA. Clin. Oral Impl. Res. (2015) 27(6):668-75.

P. Habibovic, H. Yuan, C.M. van der Valk, G. Meijer, C.A. van Blitterswijk, K. de Groot, 3D microenvironment as essential element for osteoinduction by biomaterials, Biomaterials. 26 (2005) 3565-3575. intramembraneous bone beyond the skeletal envelope using an occlusive titanium barrier. An experimental study in the rabbit, Clin. Oral Impl. Res. 6 (1995) 67-72. 
generation beyond the skeletal envelope in the rabbit calvarium, Clin. Oral Impl. Res. 14 (2003) 455-463.

[20] T. Tamura, Y. Fukase, E. Goke, Y. Yamada, S. Sato, M. Nishiyama, et al., Threedimensional evaluation for augmented bone using guided bone regeneration, J. Periodont. Res. 40 (2005) 269-276.

[21] Y. Yamada, T. Tamura, K. Hariu, Y. Asano, S. Sato, K. Ito, Angiogenesis in newly augmented bone observed in rabbit calvarium using a titanium cap, Clin. Oral Impl. Res. 19 (2008) 1003-1009.

[22] M. Knapen, D. Gheldof, P. Drion, P. Layrolle, E. Rompen, F. Lambert, Effect of leukocyte- and platelet-rich fibrin (L-PRF) on bone regeneration: a study in rabbits, Clin Implant Dent Relat Res. 17 Suppl 1 (2015) e143-52.

[23] S. Dundar, C. Ozgur, F. Yaman, O. Cakmak, A. Saybak, I.H. Ozercan, et al., Guided bone regeneration with local zoledronic acid and titanium barrier: An experimental study, Exp Ther Med. 12 (2016) 2015-2020.

C. Gjerde, K. Mustafa, S. Hellem, M. Rojewski, H. Gjengedal, M.A. Yassin, et al., Cell therapy induced regeneration of severely atrophied mandibular bone in a clinical trial, Stem Cell Res Ther. 9 (2018) 213.

B. Flautre, M. Descamps, C. Delecourt, M.C. Blary, P. Hardouin, Porous HA ceramic for bone replacement: role of the pores and interconnections - experimental study in the rabbit, J Mater Sci Mater Med. 12 (2001) 679-682.

M.Á. Brennan, A. Renaud, J. Amiaud, M.T. Rojewski, H. Schrezenmeier, D. Heymann, et al., Pre-clinical studies of bone regeneration with human bone marrow stromal cells and biphasic calcium phosphate, Stem Cell Res Ther. 5 (2013) 114-114. 
Tables

\begin{tabular}{|c|c|c|c|c|c|}
\hline Type & $\begin{array}{l}\text { Crystalline } \\
\text { phase } \\
\text { composition }\end{array}$ & $\begin{array}{c}\text { Particle size } \\
\text { /shape }\end{array}$ & $\begin{array}{c}\text { Micro } \\
\text { porosity } \\
(<10 \mu \mathrm{m}) \\
\text { size / } \\
\text { proportion }\end{array}$ & $\begin{array}{c}\text { Inter-granular } \\
\text { spaces } \\
(>10 \mu \mathrm{m}) \\
\text { size / } \\
\text { proportion }\end{array}$ & $\begin{array}{c}\text { SSA } \\
\left(\mathrm{m}^{2} / \mathrm{g}\right)\end{array}$ \\
\hline DBBM & HAP & $\begin{array}{l}0.2-1 \mathrm{~mm} \\
\text { Irregular } \\
\text { granules }\end{array}$ & $\begin{array}{c}0.01-0.03 \mu \mathrm{m} \\
31.1 \%\end{array}$ & $\begin{array}{l}200 \mu \mathrm{m} \\
23.5 \%\end{array}$ & $87.43 \pm 0.01$ \\
\hline SBCP & $\begin{array}{c}80 \text { wt. } \% \text { CDA / } \\
20 \text { wt. } \% \\
\beta-\mathrm{TCP}\end{array}$ & $\begin{array}{l}\text { 0.2-1 mm } \\
\text { Rounded } \\
\text { particles }\end{array}$ & $\begin{array}{l}0.1 \mu \mathrm{m} \\
23.8 \%\end{array}$ & $\begin{array}{l}150 \mu \mathrm{m} \\
50.3 \%\end{array}$ & $9.86 \pm 0.02$ \\
\hline
\end{tabular}

HAP: hydroxylapatite, $\mathrm{Ca}_{10}\left(\mathrm{PO}_{4}\right) 6(\mathrm{OH})_{2}$; CDA: calcium deficient hydroxyapatite ; $\beta$-TCP:

tricalcium phosphate; $\mathrm{Ca}_{3}\left(\mathrm{PO}_{4}\right)_{2} ; \mathrm{SSA}$ : specific surface area. 
Table 2: Comparison of vertical bone regeneration in hemi-spherical cups left empty, filled with DBBM or SBCP granules on the calvaria of rats after 1 day, 4 weeks and 8 weeks as determined by micro-CT.

\begin{tabular}{|r|c|c|c|}
\hline \multirow{2}{*}{ Implantation time } & \multicolumn{3}{|c|}{ (BV+MV)/TV (\%) } \\
\cline { 2 - 4 } & Left empty cups & DBBM filled cups & SBCP filled cups \\
\hline 1 day & $0.0 \pm 0.0$ & $58.3 \pm 3.6$ & $72.4 \pm 6$ \\
\hline 4 weeks & $1.36 \pm 0.42$ & $67.0 \pm 1.4$ & $67.1 \pm 5.4$ \\
\hline 8 weeks & $2.33 \pm 2.15$ & $84.8 \pm 2.8$ & $81.8 \pm 6.1$ \\
\hline
\end{tabular}

Average \pm standard error of the mean. No statistical differences were observed in any case. 
Figure captions

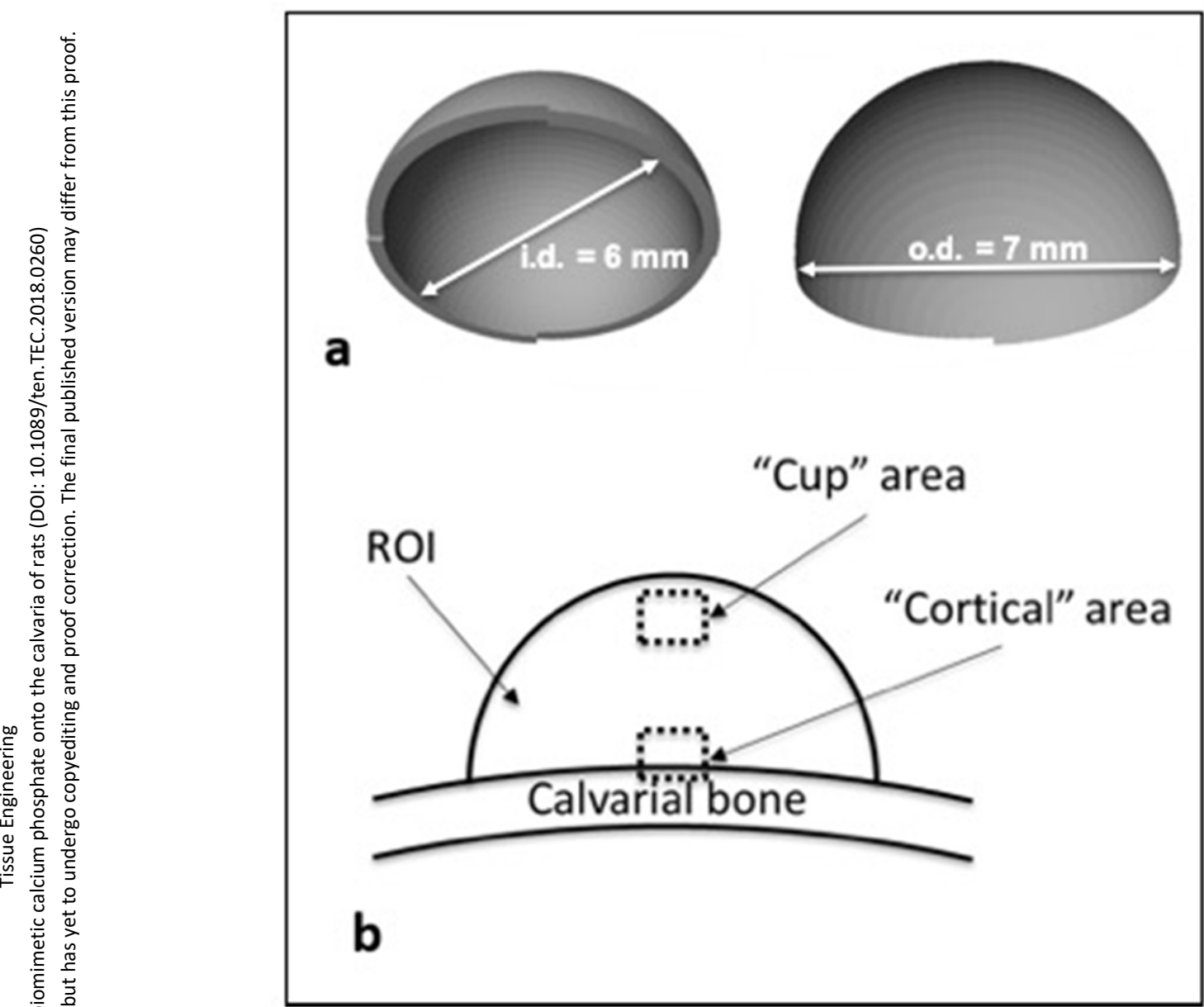

Figure 1: (a) Design of the custom PMMA cup containing the bone graft substitutes on the calvaria of rats; (b) Scheme showing the two areas of interest ("Cup" area and "Cortical" area) defined for qualitative observation and giving the region of interest (ROI) for histomorphometric analysis. 


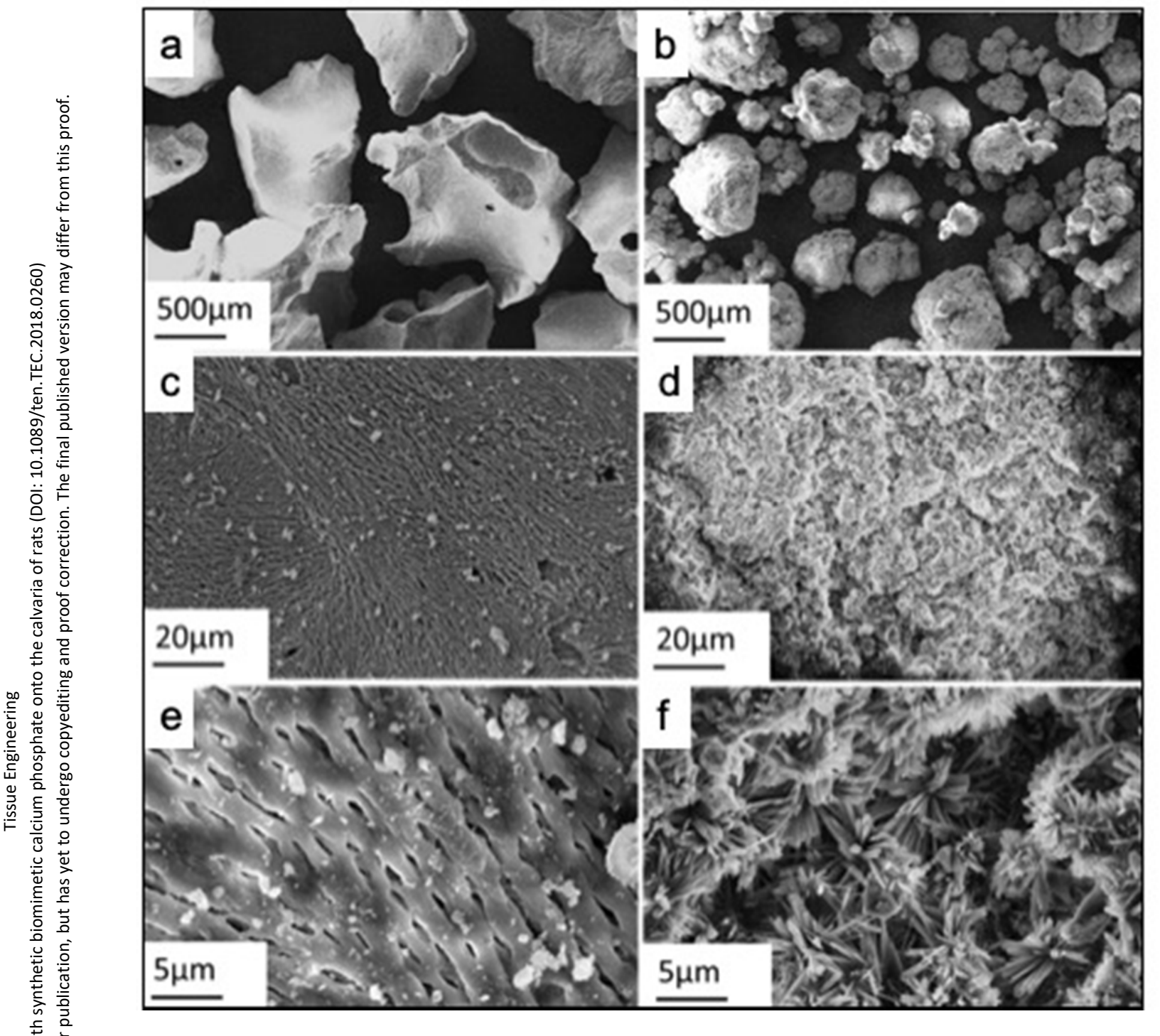

Figure 2: SEM images of DBBM (a, magnification x100; c, x 5000 and e, x10 000 ) and SBCP granules (b, x100; $d, x 1000$ and $f, x 10$ 000). 


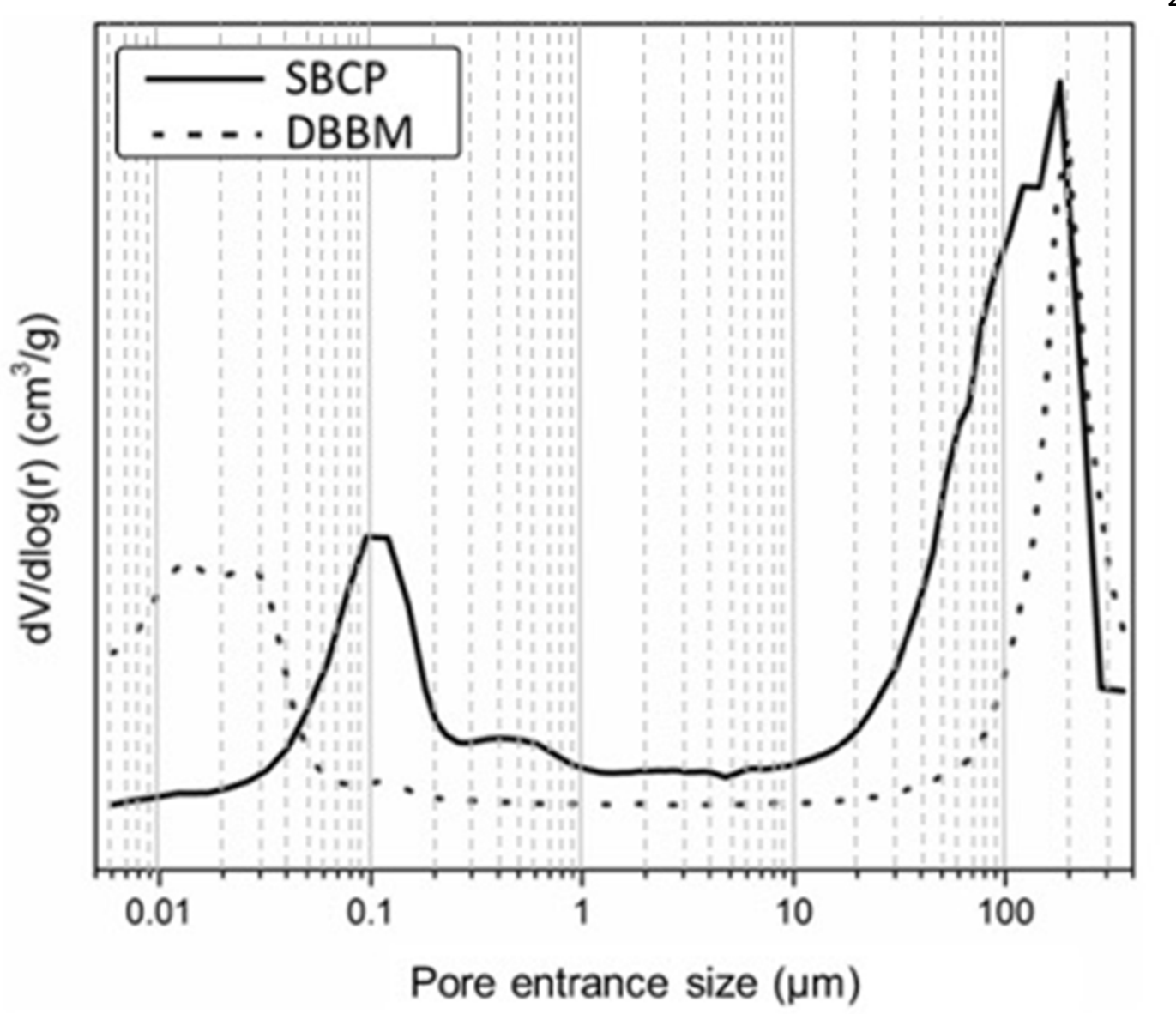

Figure 3: Mercury intrusion porosimetry of DBBM and SBCP showing intrusion volume as a function of pore entrance size. 

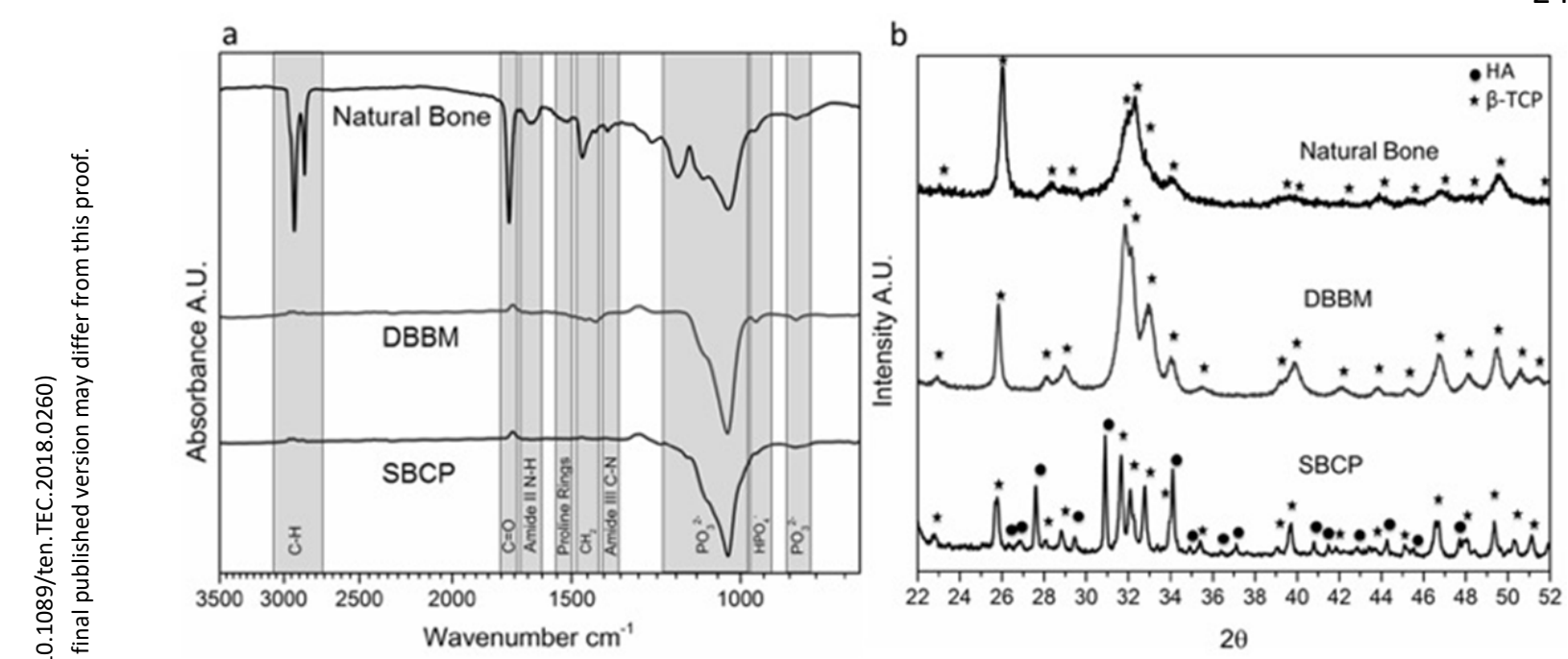

Figure 4: (a) ATR-FTIR spectra and (b) XRD patterns of natural bovine bone, DBBM and SBCP bone substitutes. Non deproteinized natural bovine bone is used in these graphs in order to qualitatively compare both biomaterials to native bone tissue composition. 


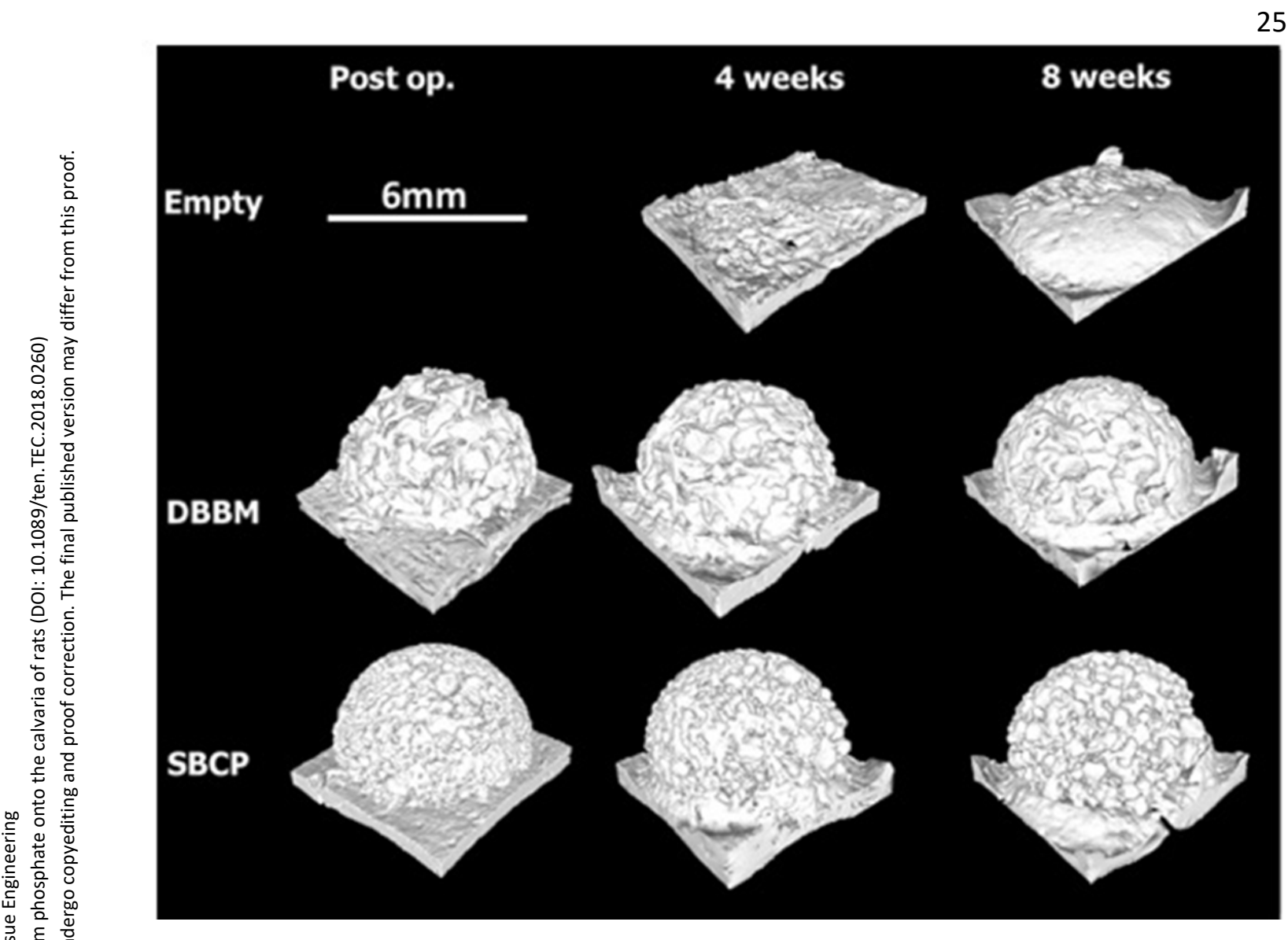

Figure 5: Micro-computed tomography reconstructions of the cups left empty, cups filled with DBBM or SBCP on the calvaria of rats at different time points: 1 day, 4 and 8 weeks post-operatively. 


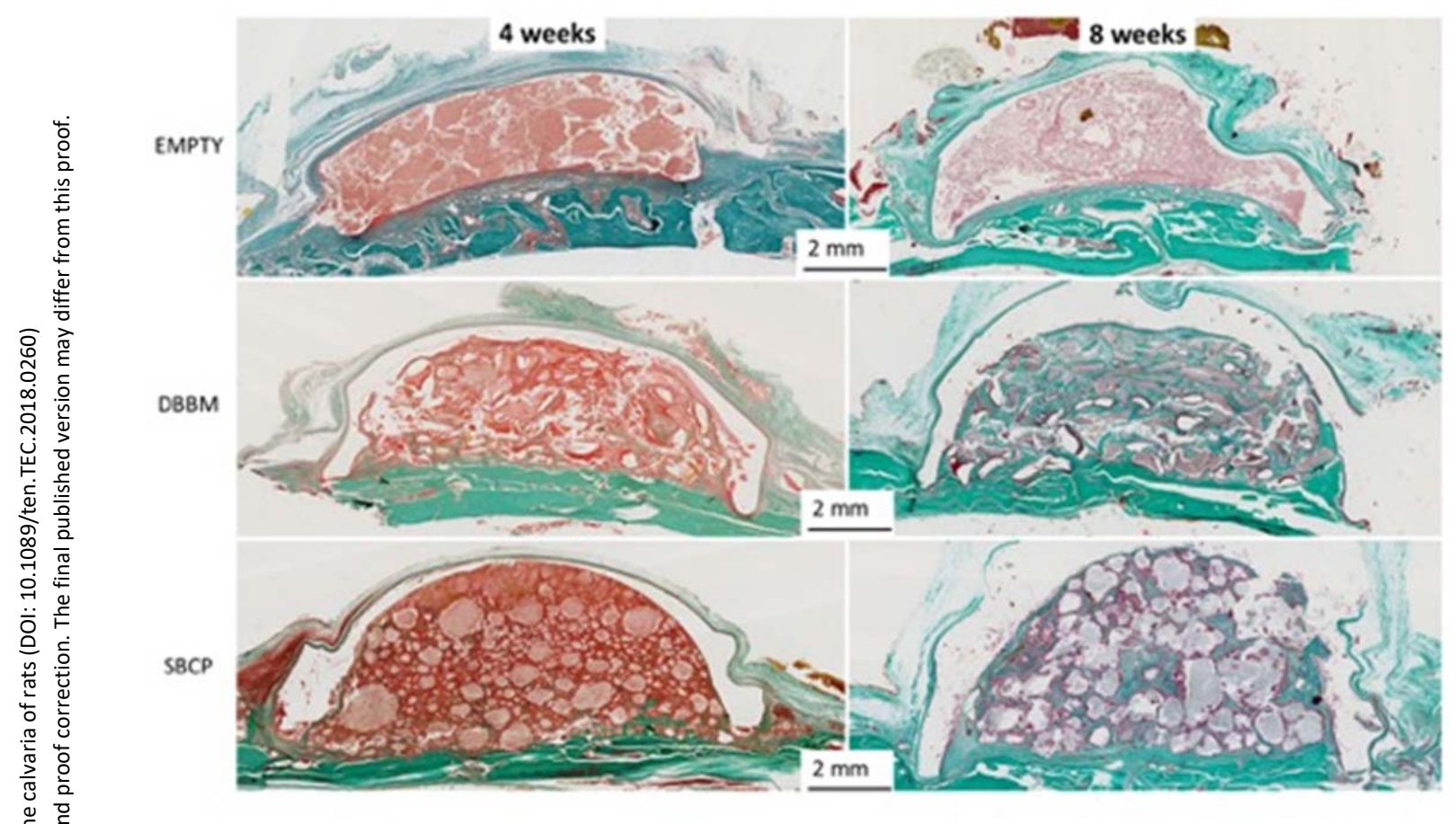

Figure 6: Histology overview of the left empty cup, cup filled with DBBM and cup filled with SBCP on the calvaria of rats after 4 weeks (first column) and 8 weeks (second column) (Masson trichrome staining, x1.5). 


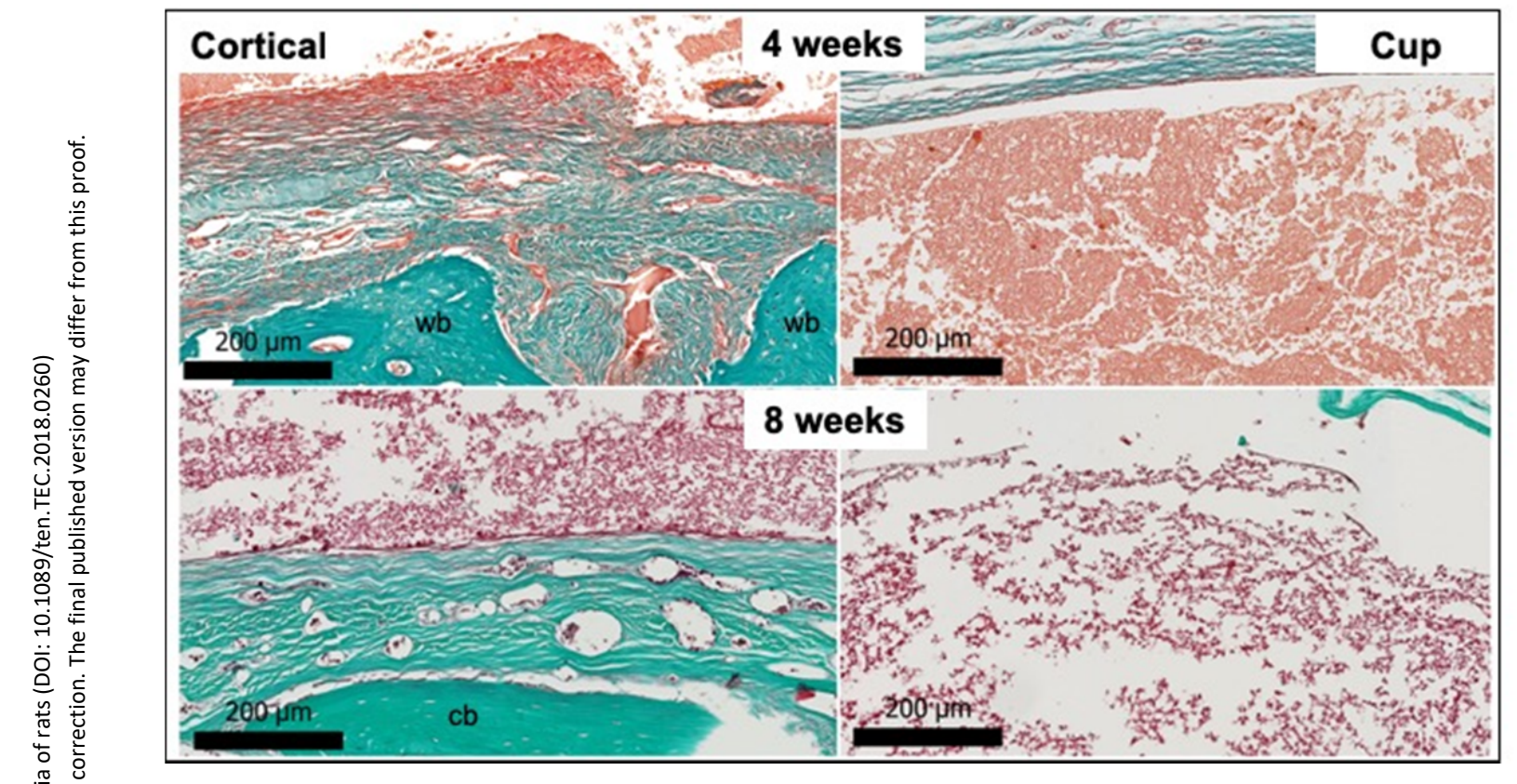

Figure 7: Histology of the left empty cup on the calvaria of rats after 4 and 8 weeks in the Cortical and Cup areas (Masson trichrome staining, x10; wb: woven bone; cb: cortical bone). 


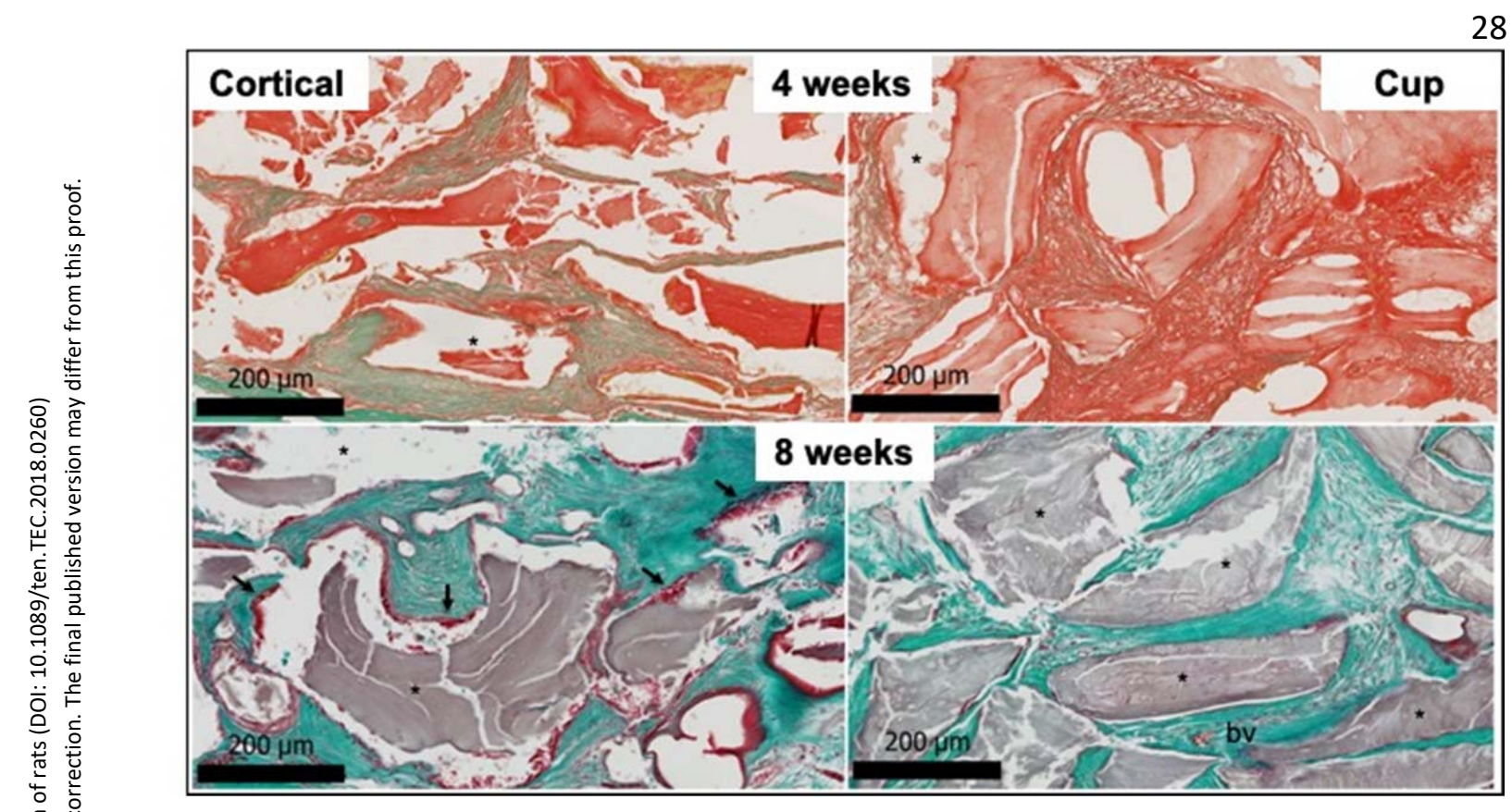

Figure 8: Histology of cups filled with DBBM after 4 and 8 weeks in the Cortical and Cup regions (Masson Trichrome staining, x10; * DBBM granules; back arrows indicate foreign body giant cells and macrophages; bv: blood vessels). 


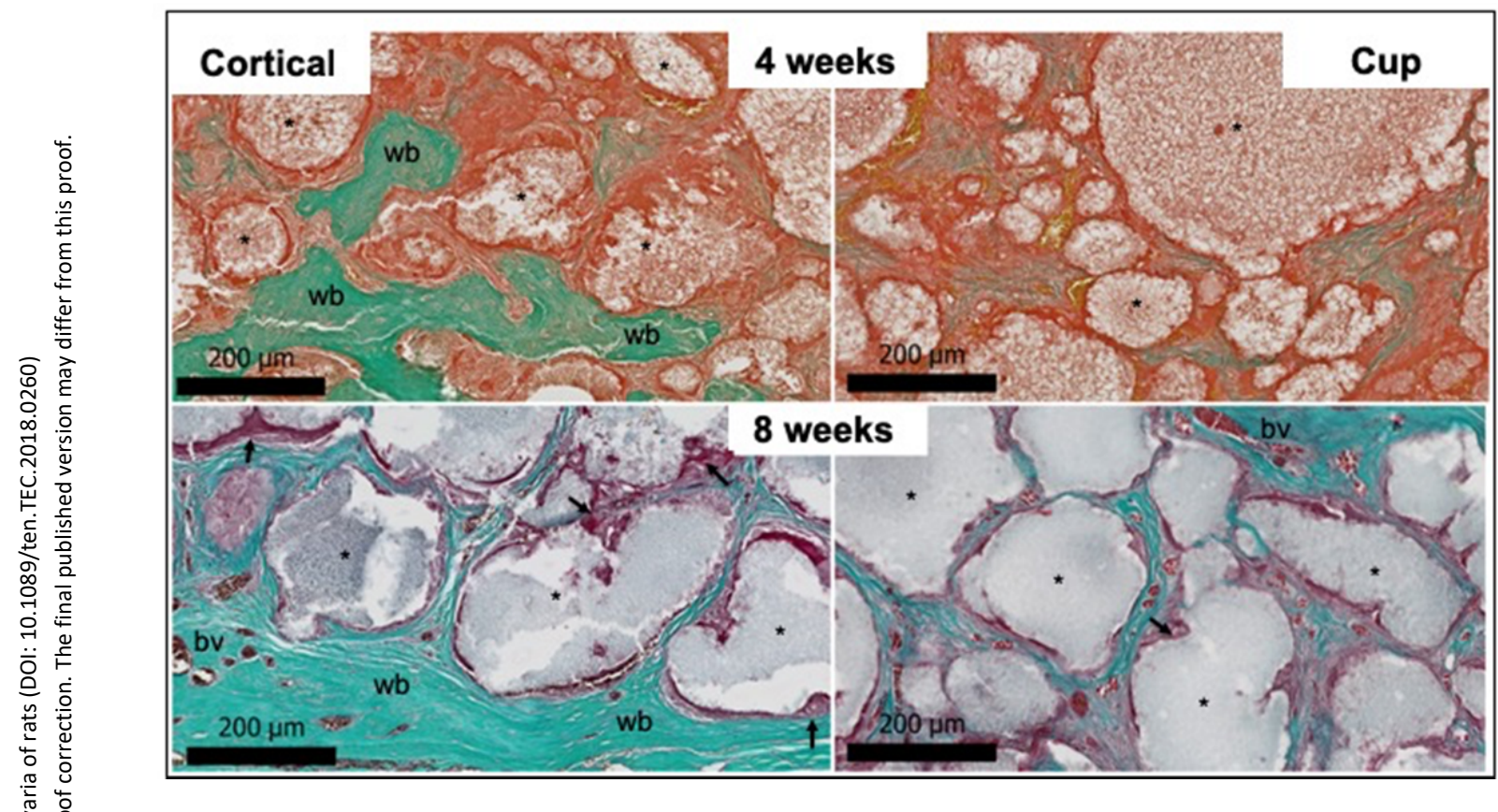

Figure 9: Histology of cups filled with SBCP after 4 and 8 weeks in the Cortical and Cup regions (Masson Trichrome staining, x10; wb: woven bone; * SBCP granules; back arrows indicates foreign body giant cells and macrophages; bv: blood vessels). 

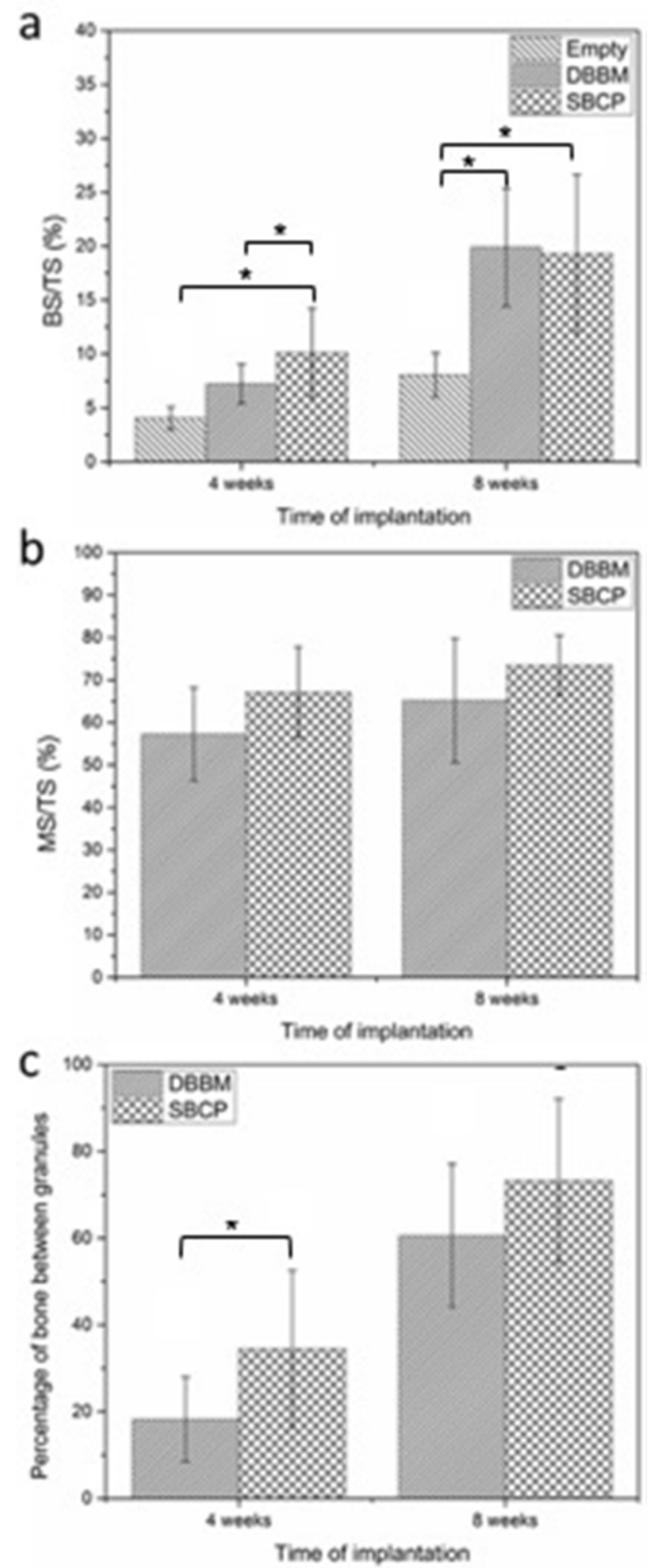

Figure 10: Histomorphometric results of (a) newly formed bone in the cortical area of the cup (BS/TS), (b) bio-material surface in the cortical area of the cup (MS/TS) (c) percentage of bone between biomaterial granules in the cortical area of the cups left empty or filled with DBBM or SBCP on the calvaria of rats after 4 and 8 weeks (* indicates p values $<0.05$ ). 